\title{
Assessment of the concentration of selected metalloproteinases (MMP-2, MMP-3, MMP-9 and MMP-13) in patients with ulcers as a complication of type 2 diabetes
}

\author{
Daria Kupczyk ${ }^{1}$, Rafał Bilski ${ }^{1}$, Renata Studzińska², Alina Woźniak
}

1Department of Medical Biology and Biochemistry Ludwik Rydygier Collegium Medicum in Bydgoszcz, Nicolaus Copernicus University in Torun, Poland

2Department of Organic Chemistry Ludwik Rydygier Collegium Medicum in Bydgoszcz, Nicolaus Copernicus University in Torun, Poland Adv Dermatol Allergol 2022; XXXIX (1): 59-65 DOI: https://doi.org/10.5114/ada.2020.100737

\begin{abstract}
Introduction: Impaired chronic wound healing is a great challenge for modern medicine. This process causes ulceration especially in the course of diseases such as type II diabetes mellitus.

Aim: This study assesses the concentration of selected matrix metalloproteinases in the example of metalloproteinase 2, 3, 9, 13 in patients with impaired healing of chronic wounds as a complication of type 2 diabetes.

Material and methods: Nineteen people took part in the assessment of wound healing in patients with type 2 diabetes. The control group consisted of 21 healthy people. In the blood serum the concentration of MMP-2, MMP-3, MMP-9 and MMP-13 was determined.

Results: The concentrations of MMP-2 and MMP-3 in the group of patients with ulcers were significantly higher (61\% and $84 \%$ accordingly) compared to those in the control group without chronic wounds. No statistically significant differences in MMP-9 and MMP-13 concentrations were observed between the study and control groups. Conclusions: The increase in MMP-2 concentration, which is particularly active in the degradation of type IV collagen, which is the main component of the basal membranes, in patients with type 2 diabetes may impede and delay the healing of chronic wounds and thus contribute to the intensification of vascular complications. In turn, the increase in MMP-3 concentration, which plays a significant role in vascular diseases, in patients with type 2 diabetes may lead to intensification of atherosclerotic changes involving the arteries of the lower extremities and ulceration.

Key words: matrix metalloproteinases, type 2 diabetes, complications of type 2 diabetes, impaired wound healing, ulcers.
\end{abstract}

\section{Introduction}

Diabetes is one of the most common metabolic disorders. The number of diabetics is constantly increasing. It is estimated that in 2025 it will reach 333 million [1] Genetic, environmental and lifestyle factors underlie its development. The cause of type 2 diabetes is impaired insulin secretion and function, leading to the development of insulin resistance [2, 3]. The group of risk factors responsible for its occurrence includes obesity, improper diet, low physical activity, genetic burden or age above 45 years [4]. In type 2 diabetes, as a result of elevated glucose levels, in addition to the occurrence of acute metabolic disorders, there are complications in the large and small vessels of a micro- and macroangiopathy nature $[5,6]$. One of the most serious is diabetic foot syndrome. During the whole life of diabetics, there is increasing risk of developing ulceration and amputation of the lower extremities, which in most cases is preceded by ulceration, which is easily infected and leads to hospitalization [7-10]. Diabetic neuropathy and ischemia of the lower extremities are the most important etiological factors of foot ulcers. In patients, sensory nerve fibers are damaged, leading to the inability to feel pain. As a result, even smaller injuries or cuts, without giving any pain, can

Address for correspondence: Daria Kupczyk, Department of Medical Biology and Biochemistry Ludwik Rydygier Collegium Medicum, Nicolaus Copernicus University, 24 Karlowicza St, 85-092 Bydgoszcz, Poland, e-mail: dariak@cm.umk.pl Received: 24.08.2020, accepted: 15.09 .2020 . 
remain unnoticed for a long time. In turn, as a result of atherosclerotic changes that arise in the vessels of the lower extremities, ischemia occurs, causing a deterioration of the healing process, fighting infection or hindering the penetration of the antibiotic to the site of infection [1]. In addition, T-lymphocyte dysfunction and immune disorders impede the healing process, thereby increasing the risk of limb amputation in diabetic patients with foot ulceration [11, 12].

Factors that may promote diabetes and are involved in the pathogenesis of associated vascular changes and complications include matrix metalloproteinases. They constitute a group of 20 (approximately) zinc-dependent proteolytic enzymes, the production of which takes place in most connective tissue cells, macrophages, leukocytes, vascular endothelial cells or cancer cells [13]. Most members of the metalloproteinase family are organized into three main and distinct domains: the N-terminal propeptide, the catalytic domain, and the hemopexin domain at the carboxy terminus of the molecule. Matrix metalloproteinases include: collagenase, matrilysin, stromelysin, gelatinase, membrane metalloproteinases and others, which have not been assigned to any of the listed groups [14]. Their main role is the degradation of extracellular matrix proteins, i.e. collagen, laminin, fibronectin and proteoglycans, which promotes cell migration and release of growth factors acting on the cells. Substrates of matrix metalloproteinases include cytokines, chemokines, growth factors and hormones, which may be activated or inactivated or transformed into products with new biological activity due to proteolytic processes [15]. Metalloproteinases are involved in the regulation of embryogenesis, control of angiogenesis, immunological processes as well as in wound healing under physiological conditions [16, 17]. However, excessive expression of the same enzymes causes that they are also involved in numerous pathological processes, leading to degradation of the extracellular matrix [18]. Excessive activation of metalloproteinases has observed in atherosclerosis or cardiovascular diseases. They also play an important role in the development and severity of vascular complications in patients with type 2 diabetes [19].

The above information shows that the care of patients with difficult to heal wounds and ulcers, which are a complication of type 2 diabetes, should be interdisciplinary. Metalloproteinases, through their ability to degrade insulin, can exacerbate the disease. The mechanism of development of these complications is currently the subject of our research.

\section{Aim}

The aim of this study is to assess the concentration of selected matrix metalloproteinases in patients with ulcers that are a complication of type 2 diabetes.

\section{Material and methods}

The study included a group of 19 people, including 10 women and 9 men, aged 68-87. The study group consisted of patients from a specialist surgery whose activities include surgical nursing and chronic wound treatment. Patients with ulceration in the foot and lower leg area, a complication of type 2 diabetes, who gave written consent for the examination, were qualified for the study. Exclusion criteria for the study included: no written consent, cancer, concomitant inflammatory diseases of the osteoarticular system, heart disease, dementia, Alzheimer's and Parkinson's disease. Patients who came to the office were under the specialist care of medical staff, because the process of chronic wound and ulcer treatment is long and requires taking a number of (actions, such as wound development, the use of total contact cast (TCC) fixations, negative pressure wound therapy (NPWT) or the use of devices and devices enabling fast and accurate transdermal measurement of oxygen partial pressure $\left(\mathrm{TcpO}_{2}\right)$ or measurement of systolic blood pressure on the fingers of the lower and upper limbs. The control group consisted of 21 healthy people aged between 32 and 62 (12 women and 9 men). The control group was selected from people without chronic diseases, without signs of inflammation or neurodegenerative processes that are characteristic for old age.

The material for biochemical tests was venous blood collected in the amount of approx. $5 \mathrm{ml}$ from the ulnar vein into tubes devoid of anticoagulant. Blood was collected in the morning. Then the collected material was transported to the Medical Biology and Biochemistry Department of the Collegium Medicum in Bydgoszcz at the Nicolaus Copernicus University in Torun. The study was permitted by the bioethics committee of Nicolaus Copernicus University in Torun (permission number KB 344/2018). Serum was obtained from blood collected in tubes without anticoagulant by centrifuging the material for $5 \mathrm{~min}$ at $5000 \times \mathrm{g}$, and then transferred to Eppendorf tubes and frozen at $-80^{\circ} \mathrm{C}$. The serum prepared in this manner was stored for the determination of metalloproteinase 2 (MMP-2), metalloproteinase 3 (MMP-3), metalloproteinase 9 (MMP-9) and metalloproteinase 13 (MMP-13). The analysis of selected metalloproteinases was carried out by the ELISA method using Cloud Clone Corp. kits. (MMP-2 cat No: SEA100Hu; MMP-3 cat No: SEA101Hu; MMP-9 cat No: SEA553Hu; MMP-13 cat No: SEA099Hu).

\section{Statistical analysis}

Statistical analysis of biochemical parameters was performed using the Statistica 10.0 program. The distribution normality was first tested using the Shapiro-Wilk test. The tested parameters met the criteria of normal distribution. The $T$ test for independent variables was used for further analysis. Next, Spearman's $R$ correlation analysis was performed. 


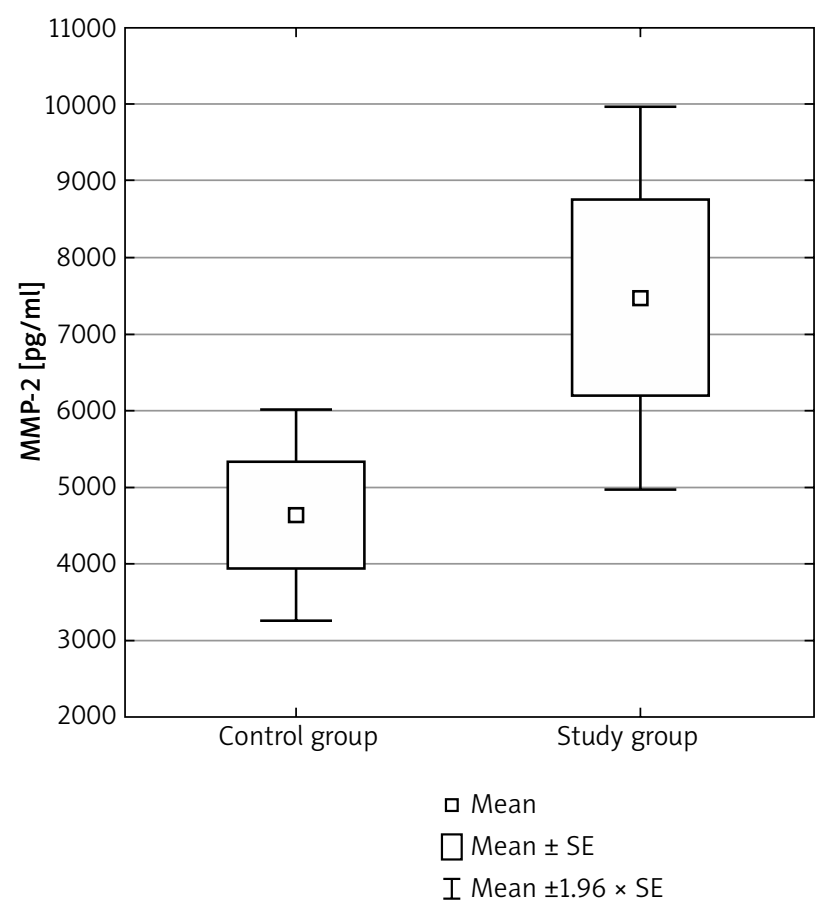

Figure 1. MMP-2 concentration in study and control group

\section{Results}

The average concentration of MMP- 2 in the serum of subjects in the study group was $61 \%$ higher than in the control group (Figure 1). The level of statistical significance was $p=0.046$. A statistically significant difference in MMP-3 concentration between groups was also observed. MMP-3 concentration in the study group was $84 \%$ higher than in the control group ( $p=0.023$ ) (Figure 2). There was no statistically significant difference in MMP-9 concentration between the study and control group ( $p=0.598)$. There was also no statistically significant difference in MMP-13 concentration between the study and control group ( $p=0.106)$ (Table 1$)$. A statistically significant positive correlation $(r=0.60, p=0.02)$ between concentrations of MMP-2 and MMP-3 was observed (Figure 3).

\section{Discussion}

Type 2 diabetes, in addition to factors such as hypertension, abdominal obesity, lipid disorders, age, smok-

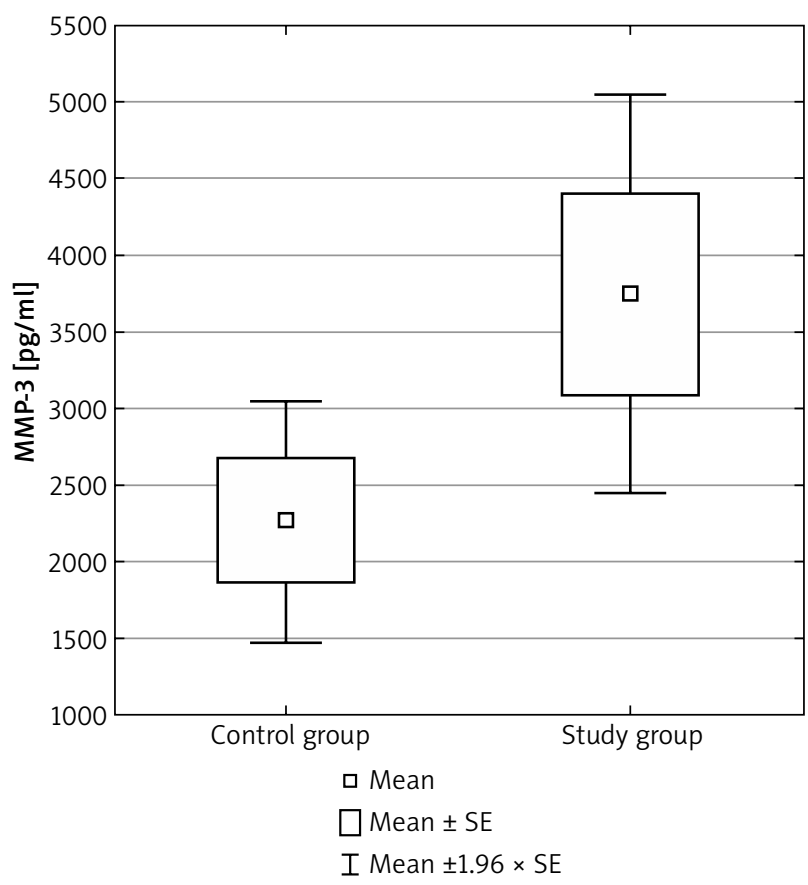

Figure 2. MMP-3 concentration in study and control group

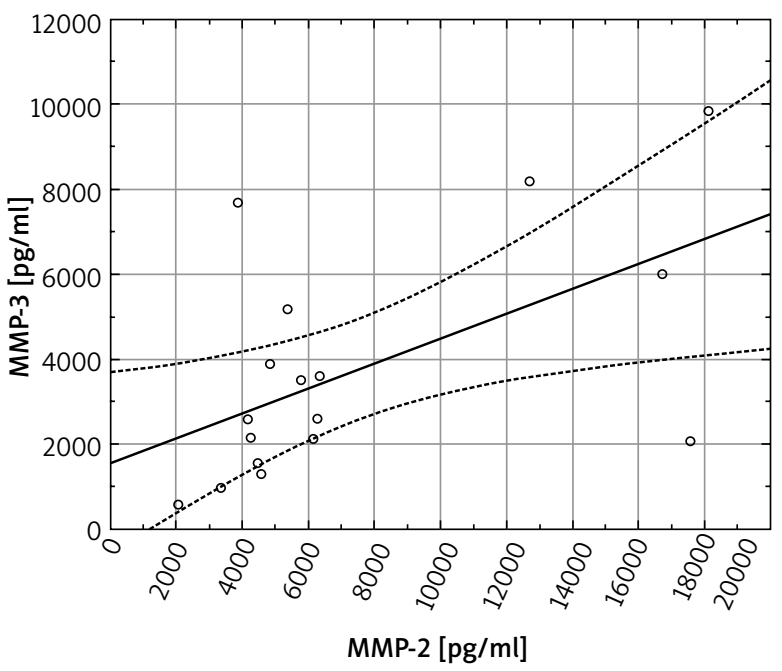

Figure 3. Linear regression of MMP3 concentration vS MMP2 concentration in the serum of subjects in the study group $(r=0.56, p=0.02)$

Table 1. Presentation of average concentration values of metalloproteinases in the study and control groups

\begin{tabular}{|c|c|c|c|c|c|}
\hline \multirow[t]{2}{*}{ Parameter } & \multicolumn{2}{|c|}{ Study group $(n=19)$} & \multicolumn{2}{|c|}{ Control group $(n=21)$} & \multirow[t]{2}{*}{$P$-value } \\
\hline & Mean & Standard deviation & Mean & Standard deviation & \\
\hline MMP-2 [pg/ml] & 7463.9 & 5275.6 & 4634.3 & 3302.1 & 0.046 \\
\hline MMP-3 [pg/ml] & 3745.0 & 2723.6 & 2030.6 & 1582.4 & 0.023 \\
\hline MMP-9 [pg/ml] & 2289.4 & 355.6 & 2461.1 & 404.2 & 0.598 \\
\hline MMP-13 [pg/ml] & 829.9 & 1180.7 & 1171.3 & 1750.0 & 0.106 \\
\hline
\end{tabular}


ing and hyperhomocysteinemia, is one of the risk factors for cardiovascular disease [20]. It can also lead to kidney failure, blindness, and in extreme cases to amputation of the lower limbs. The above-mentioned factors act continuously and progressively on the wall of blood vessels, leading to its damage and impairment of vascular endothelial function, by initiating the inflammatory process [21]. As a result, endothelial cells secrete a number of substances involved, among other things, in inflammatory processes, coagulation or fibrinolysis. Endothelial dysfunction, along with chronic hyperglycemia, underlies the development of micro- and macroangiopathy complications. These complications relate to the remodeling of the vascular wall. In microangiopathy complications, there are changes in the middle membrane of small and medium arteries, while in macroangiopathic complications, pathological changes affect the atherosclerotic capsule. One of the stages that initiate the process of remodeling blood vessels, resulting from damage to the endothelium, is the degradation of their basal membrane and extracellular matrix. The main component of the basal membrane is type IV collagen.

In relation to this collagen, MMP-2 and MMP-9 metalloproteinases show high substrate activity. They are present in the wall of blood vessels and come from T lymphocytes and smooth muscle cells. In addition, MMP-9 can also be produced by macrophages, mast cells, fibroblasts and endothelial cells. The effect of these metalloproteinases is not limited to degradation of type IV collagen, as they also affect the processes of apoptosis, cytokine release or maintaining tissue continuity [22, 23]. A major role is assigned to these metalloproteinases in the development of atherosclerosis, especially in the degradation of collagen, glycosaminoglycans and proteoglycans $[24,25]$. Research from recent years also indicates that a group of matrix metalloproteinases belonging to type IV collagenases is involved in the development of autoimmune diseases such as type 1 diabetes, but also type 2 diabetes. Elevated serum glucose induces expression and activity of MMP-2 collagenase in endothelial cells and MMP-9 in macrophages [26]. Gharagozlian et al. found that the activity of MMP-2 and MMP-9 in the serum of patients with type 1 diabetes was significantly higher compared to the control group [27]. Similarly, Shiau et al. found that the concentration of both type IV collagenases is higher in diabetic patients without complications compared to the control group [28]. Experiments carried out by Maxwell et al. did not indicate an increase in MMP-2 plasma concentration in patients with type 1 diabetes, but they observed an increase in MMP-9 in these patients compared to the control group [29]. In turn, Prystupa et al. reported that the level of MMP-2 activity was reduced in patients with type 2 diabetes compared to the control group, while MMP-9 activity did not differ statistically from the control group [30].
Our studies also showed no statistically significant differences in MMP-9 activity in patients with ulcers that are a complication of type 2 diabetes compared to the control group. The concentration of MMP-9 for both patients and the control group was in a similar range (from 2289.41 to $2765.40 \mathrm{pg} / \mathrm{ml}$ for patients and from 2461.06 to $2866.4 \mathrm{pg} / \mathrm{ml}$ for the control group, respectively). In contrast, the concentration of MMP-2 observed in patients with ulcers was clearly higher and ranged from 2081.80 to $18124.65 \mathrm{pg} / \mathrm{ml}$, compared to the control group (617.25 to $14936.15 \mathrm{pg} / \mathrm{ml}$ ).

When selecting patients for the clinical trial, we tried to obtain a homogeneous group of patients only with ulcers that are a complication of type 2 diabetes, as additional disorders could affect the concentration of metalloproteinases in the blood serum. For example, when assessing MMP-9 concentration, the presence of hyperhomocysteinemia in patients should be taken into account, as high levels of this amino acid are reported to be one of the factors that increase MMP-9 levels. Moshal et al. demonstrated in their research the relationship between hyperhomocysteinemia and gene expression for MMP-9 in endothelial cells [30]. It can therefore be presumed that in patients with complications of type 2 diabetes, hyperhomocysteinemia may cause an increase in MMP-9 production in vascular endothelial cells. In addition, factors such as obesity or hypertriglyceridemia, among others, may increase MMP-9 activity in type 2 diabetes [30]. More importantly, in the context of the role of MMP-9, it seems that this metalloproteinase is important for maintaining the correct tone of the blood vessel wall [31]. In addition, Salonurmi et al. conducted an experiment on transgenic mice, based on which they verified the role of MMP-9 in wound healing. In these mice, a fragment of human DNA encoding TIMP-1 was placed in front of the promoter of the gene encoding MMP-9. As a result, TIMP-1 was expressed in transgenic mice in tissues in which wild mice were expressed. Studies on this type of mice have shown that the wound healing process resulting from sterile dorsal incisions is significantly delayed [32]. In turn, Frøssing et al. found no effect of MMP-2 on wound healing in plasminogen-deficient mice [33]. Another metalloproteinase whose concentration was evaluated in this study is MMP-3, which belongs to the stromelysin group. It has high proteolytic activity. In addition to the hydrolysis of components of the extracellular matrix, it activates numerous zymogens of metalloproteinases (proMMP). Its presence is also necessary for the activation of MMP-1 [23, 34]. Amanzadeh et al. observed lower activity of MMP-3 in patients with macroalbuminuria in comparison with patients with microalbuminuria and normoalbuminuria. In addition, the research team found a positive correlation between glomerular filtration rate and MMP-3 activity in diabetic patients. On this basis, it can be concluded that MMP-3 may play a role in the pathogen- 
esis and development of diabetic nephropathy [35]. Zákovičová et al. observed higher levels of MMP-3 and MMP-9 in patients with type 2 diabetes without cardiovascular complications compared to the control group [36]. They also found that the increase in MMP-3 was correlated with albuminuria. Our own study showed a statistically significantly higher MMP-3 concentration in patients with ulcers in the course of type 2 diabetes compared to the control group. The concentration of MMP-3 in patients ranged from 575.20 to $9824.85 \mathrm{pg} / \mathrm{ml}$, while in the control group lower levels of this metalloproteinase (in the range 355.10-7061.65 pg/ml) were observed.

Baugh et al. rated participation of, among other factors, MMP-3 and MMP-9 in the process of coronary plaque rupture as a result of hyperglycemia associated with type 2 diabetes. However, they did not observe significant differences in levels of MMP-3 or MMP-9 between the type 2 diabetes group without previous cardiovascular complications and the control group [37]. Peeters et al. observed, among other observations, a higher level of MMP-3 in patients with albuminuria in the course of type 1 diabetes, which may indicate participation in the pathogenesis of complications of this type of diabetes due to disorders in the regulation of extracellular matrix remodeling [19]. Knaś et al. detected higher MMP-3 activity in the skin of rats with insulin resistance in type 1 diabetes compared to the control group [38]. Available literature data indicate the involvement of MMP-3 in the pathogenesis of nephropathy complications in the course of type 1 diabetes. The sparsity of data from the literature and promising results of our own research encourage continuation of studies on a larger number of subjects. Research can then be extended to substrates and MMP-3 inhibitors to better understand the role of this metalloproteinase in the development of complications of type 2 diabetes in the form of ulceration - especially since, as mentioned earlier, MMP-3 is involved in the activation of MMP-1, which in turn is involved in wound healing and ulceration in the course of diabetes [38]. In addition, our own study showed a positive correlation between MMP-2 and MMP-3 in the study group with ulcers in the course of type 2 diabetes, which may indicate the simultaneous secretion of both metalloproteinases in complications of type 2 diabetes, leading to the formation of difficult to heal wounds and ulcers (Figure 3).

This study also assessed the concentration of collagenase 3, which is metalloproteinase 13 (MMP-13). However, no statistically significant difference in the concentration of this metalloproteinase was found between the test group with ulcers in the course of type 2 diabetes and the control group. MMP-13 concentration for both patients and the control group was in a similar range ( 829.92 to $4254.03 \mathrm{pg} / \mathrm{ml}$ for patients and 1171.29 to $6352.4 \mathrm{pg} / \mathrm{ml}$ for the control group). However, a focal increase in MMP-13 expression is observed in atherosclerosis [39]. Analysis of histopathological materials obtained from atherosclerotic lesions indicates that the presence of MMP-13 is characteristic for all types of atherosclerotic plaques. Increased MMP-13 activity was observed in inflammatory and lipid-rich atherosclerotic lesions compared to fibrous lesions $[40,41]$. The main sources of metalloproteinases in atherosclerotic plaques are macrophages and foam cells formed from them. It is the activation of macrophages with the participation of proinflammatory cytokines that significantly increases the expression and release of metalloproteinases, including MMP-13, causing degradation of the extracellular matrix [42-44]. In diabetes, which may be accompanied by a number of changes, such as dyslipidemia or atherosclerosis, the processes of nonenzymatic glycation of proteins intensify, resulting in advanced (end) glycation products, called advanced glycation end-products. Proteins with a long half-life, e.g. collagen, undergo glycation. As a result of this process, there occurs remodeling of the basal membrane, which thickens and increases the susceptibility of blood vessels to forming clots [45]. The paucity of literature data and promising results of our own research justify continuation of research with the participation of a larger group of respondents. Since obesity, high cholesterol and lipid levels are among the risk factors for atherosclerosis, one can try to find an element connecting this disease unit with diabetes, which also increases lipid oxidation. Endothelial dysfunction characteristic of the diseases described above leads to the degradation of the extracellular matrix, which may result in increased secretion of metalloproteinases. In patients with type 2 diabetes, the cause of the deterioration of quality of life is development of vascular complications. Metalloproteinases are involved in the pathogenesis of diabetes as well as in the development and progression of related complications. They can also exacerbate the course of the disease. The degradation of basal membranes, components of the extracellular matrix dependent on metalloproteinases, underlies the development of diabetic complications.

\section{Conclusions}

Therefore, the research presented in this paper should be continued in order to collect and develop data for a larger study group.

\section{Conflict of interest}

The authors declare no conflict of interest.

\section{References}

1. Borissova AM, Shinkov A, Kovatcheva R, et al. Changes in the prevalence of diabetes mellitus in Bulgaria (20062012). Clin Med Insights Endocrinol Diabetes 2015; 8: 41-5.

2. Abdul-Ghani MA, Tripathy D, DeFronzo RA. Contributions of beta-cell dysfunction and insulin resistance to the patho- 
genesis of impaired glucose tolerance and impaired fasting glucose. Diabetes Care 2006; 29: 1130-9.

3. Del Prato S. Role of glucotoxicity and lipotoxicity in the pathophysiology of type 2 diabetes mellitus and emerging treatment strategies. Diabet Med 2009; 26: 1185-92.

4. Tripathi BK, Srivastava AK. Diabetes mellitus: complications and therapeutics. Med Sci Monit 2006; 12: 130-47.

5. Genuth S, Eastman R, Kahn R, et al.; American Diabetes Association (2000). Implications of the United Kingdom Prospective Diabetes Study. Diabetes Care 2003; 26 Suppl 1: S28-32; Suppl 1: 27-31.

6. Jung KH, Chu K, Lee ST, et al. Risk of macrovascular complications in type 2 diabetes mellitus: endothelial microparticle profiles. Cerebrovasc Dis 2011; 31: 485-93.

7. Singh N, Armstrong DG, Lipsky BA. Preventing foot ulcers in patients with diabetes JAMA 2005; 293: 217-28.

8. Lavery LA, Ashry HR, van Houtum W, et al. Variation in the incidence and proportion of diabetes related amputations in minorities. Diabetes Care 1996; 19: 48-52.

9. Lavery LA, van Houtum W, Astry HR, et al. Diabetes related lower extremity amputations disproportionately affect blacks and Mexican Americans. South Med J 1999; 92: 593-9.

10. Pecoraro RE, Reiber GE, Burgess EM. Pathways to diabetic limb amputations: basis for prevention. Diabetes Care 1990; 13: 513-21.

11. Geerlings SE, Hoepelman Al. Immune dysfunction in patients with diabetes mellitus. FEMS Immunol Med Microbiol 1999; 26: 259-65.

12. Blakytny $R$, Jude $E$. The molecular biology of chronic wounds and delayed healing in diabetes. Diabet Med 2006; 23: 594 608.

13. Haq M, Shaeii AE, Zervos EE, Rosemurgy AS. In vitro and in vivo matrix metalloproteinase production by pancreatic cancer cells and by distant organs. Int J Surg Invest 2000; 1: 459-65.

14. Laronha $\mathrm{H}$, Caldeira J. Structure and function of human matrix metalloproteinases. Cells 2020; 9: 1076.

15. Jabłońska-Trypuć A, Matejczyk M, Rosochacki S. Matrix metalloproteinases (MMPs), the main extracellular matrix (ECM) enzymes in collagen degradation, as a target for anticancer drugs. J Enzyme Inhib Med Chem 2016; 31 (Suppl 1): 177-83.

16. Salmela MT, Pender SL, Karjalainen-Lindsberg ML, et al. Collagenase-1 (MMP-1), matrilysin-1 (MMP-7), and stromelysin-2 (MMP-10) are expressed by migrating enterocytes during intestinal wound healing. Scand J Gastroenterol 2004; 39: 1095-104

17. Egeblad M, Werb Z. New functions for the matrix metalloproteinases In cancer progression. Nat Rev Cancer 2002; 2 163-76.

18. Mittal R, Patel AP, Debs LH, et al. Intricate functions of matrix metalloproteinases in physiological and pathologica conditions. J Cell Physiol 2016; 231: 2599-621.

19. Peeters SA, Engelen L, Buijs J, et al. EURODIAB Prospective Complications Study Group. Plasma levels of matrix metalloproteinase-2, -3, -10, and tissue inhibitor of metalloproteinase- 1 are associated with vascular complications in patients with type 1 diabetes: the EURODIAB Prospective Complica tions Study. Cardiovasc Diabetol 2015; 14: 31.

20. Brunner $H$, Cockcroft JR, Deanfield J, et al. Endothelial function and dysfunction. Part II: Association with cardiovascular risk factors and diseases: a statement by the Working Group on Endothelins and Endothelial Factors of the European Society of Hypertension. J Hypertens 2005; 23: 233-46.
21. Schalkwijk CG, Stehouwer CD. Vascular complications in diabetes mellitus: the role of endothelial dysfunction. Clin Sci 2005; 109: 143-59.

22. Flamant M, Placier S, Dubroca C, et al. Role of matrix metalloproteinases in early hypertensive vascular remodeling. Hypertension 2007; 50: 212-8.

23. Visse R, Nagase H. Matrix metalloproteinases and tissue inhibitors of metalloproteinases: structure, function, and biochemistry. Circ Res 2003; 92: 827-39.

24. Fic P, Zakrocka I, Kurzepa J, Stepulak A. Metaloproteinazy w miażdżycy naczyń krwionośnych. Post Hig Med Dosw 2011; 65: 16-27.

25. Lottus JM, Naylor AR, Bell PRF. Matrix metalloproteinases and atherosclerotic plaque instability. Br J Surg 2002; 89: 680-94.

26. Nagase H, Woessner JF. Matrix metalloproteinases. J Biol Chem 1999; 274: 21491-4.

27. Gharagozlian S, Svennevig K, Bangstad HJ, et al. Matrix metalloproteinases in subjects with type I diabetes. BMC Clin Pathol 2009; 9: 7-11.

28. Shiau MY, Tsai ST, Tsai KJ, et al. Increased circulatory MMP-2 and MMP-9 levels and activities in patients with type 1 diabetes mellitus. Mt Sinai J Med 2006; 73: 1024-8.

29. Maxwell PR, Timms PM, Chandran S, Gordon D. Peripheral blood level alterations of TIMP-1, MMP-2 and MMP-9 in patients with type 1 diabetes. Diabet Med 2001; 18: 777-80.

30. Moshal KS, Sen U, Tyagi N, et al. Regulation of homocysteine-induced MMP-9 by ERK1/2 pathway. Am J Physiol Cell Physiol 2006; 290: 883-91.

31. Su J, Palen DI, Lucchesi PA, Matrougui K. Mice lacking the gene encoding for MMP-9 and resistance artery reactivity. Biochem Biophys Res Commun 2006; 349: 1177-81.

32. Salonurmi T, Parikka M, Kontusaari S, et al. Overexpression of TIMP-1 under the MMP-9 promoter interferes with wound healing in transgenic mice. Cell Tissue Res 2004; 315: 27-37.

33. Frøssing S, Rønø B, Hald A, et al. Skin wound healing in MMP2-deficient and MMP2/plasminogen double-deficient mice. Exp Dermatol 2010; 19: 234-40.

34. Suzuki K, Enghild JJ, Morodomi T, et al. Mechanisms of activation of tissue procollagenase by matrix metalloproteinase 3 (stromelysin). Biochemistry 1990; 29: 10261-70.

35. Amanzadeh M, Mota A, Zarghami N, et al. Association between matrix metalloproteinase-3 activity and glomerular filtration rate and albuminuria status in patients with type 2 diabetes mellitus. Iran J Kidney Dis 2018; 12: 40-7.

36. Zákovičová E, Charvat J, Kukacka J, et al. Circulating serum matrix metalloproteinase- 3 and metalloproteinase- 9 are not associated with echocardiographic parameters of diastolic function in asymptomatic type 2 diabetic patients. J Int Med Res 2010; 38: 2093-9.

37. Baugh MD, Gavrilovic J, Davies IR, et al. Monocyte matrix metalloproteinase production in type 2 diabetes and controls - a cross sectional study. Cardiovasc Diabetol 2003; 2: $1-4$.

38. Knaś $M$, Wołosik A, Zalewska A, et al. The skin remodeling in type 1 diabetes and insulin resistance animal models. Phsyiol Res 2015; 64: 875-81.

39. Menghini R, Uccioli L, Vainieri E, et al. Expression of tissue inhibitor of metalloprotease 3 is reduced in ischemic but not neuropathic ulcers from patients with type 2 diabetes mellitus. Acta Diabetologica 2013; 50: 907-10.

40. Yu Y, Koike T, Kitajima S, et al. Temporal and quantitative analysis of expression of metalloproteinases (MMPs) and 
their endogenous inhibitors in atherosclerotic lesions. Histol Histopathol 2008; 23: 1503-16.

41. Newby AC. Dual role of matrix metalloproteinases (matrixins) in intimal thickening and atherosclerotic plaque rupture. Physiol Rev 2005; 85: 1-31.

42. Newby AC. Metalloproteinase expression in monocytes and macrophages and its relationship to atherosclerotic plaque instability. Arterioscler Thromb Vasc Biol 2008; 28: 2108-14.

43. Busti C, Falcinelli E, Momi S, Gresele P. Matrix metalloproteinases and peripheral arterial disease. Intern Emerg Med 2010; 5: 13-25.

44. Kupczyk D, Bilski R, Przewięźlikowski A, et al. Concentration of proinflammatory cytokines in patients with ulcers as complications of type 2 diabetes mellitus. Adv Dermatol Allergol 2020. doi:10.5114/ada.2020.96702.

45. Stowik-Żyłka D, Safranow K, Ciechanowski K. Późne produkty glikacji białek (AGE) - powstawanie, znaczenie patogenetyczne, oznaczanie. Diabetol Pol 2001; 8: 281-21. 